\title{
The Structure of the Proton as Measured at HERA
}

\author{
Andrew Mehta on behalf of the H1 and ZEUS Collaborations \\ Dept. of Physics, University of Liverpool, L69 3BX, UK.
}

\begin{abstract}
Recent measurements that help constrain the structure of the proton from the HERA $e p$ collider experiments $\mathrm{H} 1$ and ZEUS are described. Measurements of the proton structure functions $F_{2}\left(x, Q^{2}\right), x F_{3}\left(x, Q^{2}\right)$ and $F_{L}\left(x, Q^{2}\right)$ along with the $e^{+} p$ and $e^{-} p$ charged current cross sections are presented. The precision of the data is further improved by combining the measurements from the two experiments. The combined measurements are used in a QCD fit to provide a set of proton parton distribution functions that include experimental and theoretical uncertainties.
\end{abstract}

Keywords: Structure Function, DIS, QCD

PACS: $12.38 . \mathrm{Qk}, 13.60 .-\mathrm{r}$

\section{INTRODUCTION}

Deep inelastic scattering (DIS) has long been used to measure the structure of hadrons. Measurements with a fixed target helped establish the quark parton model and the theory of the strong force, quantum chromodynamics (QCD). The measurements of proton structure at HERA, particularly at very low fractional parton momentum, have yielded extremely precise parton distribution functions (PDFs), essential for making QCD predictions at present and future hadron colliders.

The HERA 1 run took place in the years 1992-2000, when HERA was operated in both $e^{+} p$ and $e^{-} p$ scattering modes at a center of mass energy in the range $\sqrt{s}=$ $300-318 \mathrm{GeV}$. Each of the experiments $\mathrm{H} 1$ and ZEUS collected about $120 \mathrm{pb}^{-1}$ of data. After a major luminosity and detector upgrade the HERA 2 run took place from 2004-2007 with polarized $e^{+}$and $e^{-}$beams. Around $380 \mathrm{pb}^{-1}$ of the data was taken $\sqrt{s}=318 \mathrm{GeV}$ and about $20 \mathrm{pb}^{-1}$ at lower beam energies of $\sqrt{s}=251 \mathrm{GeV}$ and $\sqrt{s}=225 \mathrm{GeV}$ in order to enable a measurement of the longitudinal structure function $F_{L}$.

The large data samples collected have allowed a determination of all the possible neutral current $(\mathrm{NC}, e p \rightarrow e X)$ structure functions $F_{2}, x F_{3}$ and for the first time at HERA $F_{L}$. The structure function $F_{2}$ is sensitive to all quark species and dominates the cross section throughout the accessible phase space. It is the most precisely determined structure function. The quantity $x F_{3}$ is sensitive to the valence quarks. Since the cross section only becomes sensitive to $x F_{3}$ via the exchange of the $Z^{0}$ boson, its influence is limited to the very high $Q^{2}$ electroweak regime. Finally, $F_{L}$ is sensitive to higher order gluon radiation processes providing valuable confirmation of the gluon content of the proton.

The structure functions are usually presented in terms of the kinematic variables Bjorken $x$, the fraction of the proton's momentum carried by the struck quark and $Q^{2}$ 
the negative square of the 4-momentum transfer of the exchanged boson. $Q^{2}$ may be interpreted as the resolving power of the exchange, with increasing $Q^{2}$ able to resolve smaller distances within the proton. They may be derived from the differential cross sections as

$$
\frac{\mathrm{d} \sigma_{N C}\left(e^{ \pm} p\right)\left(x, Q^{2}, y\right)}{\mathrm{d} x \mathrm{~d} Q^{2}} \simeq \frac{2 \pi \alpha^{2}}{x Q^{4}}\left[Y_{+} \tilde{F}_{2}\left(x, Q^{2}\right) \mp Y_{-} x \tilde{F}_{3}\left(x, Q^{2}\right)-y^{2} \tilde{F}_{L}\left(x, Q^{2}\right)\right]
$$

Here $\alpha$ is the electromagnetic coupling constants and $Y_{ \pm}=1 \pm(1-y)^{2}$ where $y=$ $Q^{2} / s x$.

Like the neutral current cross section, the charged current $(\mathrm{CC}, e p \rightarrow v X)$ cross section is an important tool to measure the structure of the proton, particularly since the charged current process is sensitive to the quark flavor decomposition of the proton. Measurements of the $e^{+} p$ and $e^{-} p$ scattering cross sections have allowed independent determination of the $d$ quark and $u$ densities.

\section{MEASUREMENTS AND QCD FIT}

H1 have recently published new measurements of $F_{2}$ in the region $0.2<Q^{2}<$ $150 \mathrm{GeV}^{2}$ [1]. This set of measurements contain points which reach a precision of $1.3 \%$. The data has been combined with measurements from ZEUS and data from $\mathrm{H} 1$ and ZEUS at higher $Q^{2}$ to provide the most precise measurement of $F_{2}$ at low $x$ [2]. A small selection of the combined measurements is presented in figure 1 (left) which shows the 'reduced cross section', which is approximately equal to $F_{2}$ apart from small corrections for $F_{L}$ at high $y$. The data show strong scaling violations at low $x$. The scaling violations allow the gluon density of the proton to be constrained. The data are well described by the QCD fit.

In figure 1 (right) the measurement of the CC cross section for $e^{+} p$ scattering for combined H1 and ZEUS data is shown. A similar measurement for $e^{-} p$ scattering is also available [2]. Whilst the $e^{-} p$ data are mainly sensitive to the $u$ quark density, which is well constrained by the NC data, the $e^{+} p$ data help constrain the $d$ density which is not so well determined by the NC data.

A combined H1 ZEUS measurement of $x F_{3}$ [3] has also been evaluated by taking the difference between the NC cross sections from $e^{-} p$ and $e^{+} p$ scattering (see equation1). This measurement constrains the valence PDFs of the proton.

The combined HERA NC and CC cross sections are used to extract the proton PDFs by performing an NLO QCD fit [2]. Only HERA inclusive data are used in this fit. The PDFs are parameterized at a low scale $\left(Q_{0}^{2}=1.9 \mathrm{GeV}^{2}\right)$ and are evolved using the DGLAP equations to higher $Q^{2}$. The heavy flavor quarks are treated using a variable flavor number scheme that takes the quark mass into account [4]. The $s$ quark density is fixed at the starting scale since there are no good constraints from HERA data. The fit takes into account the experimental uncertainties including correlations bin to bin and between experiments or datasets. Model uncertainties are taken into account by varying the heavy quark masses, the $s$ density, $Q_{0}$ and the minimum $Q^{2}$ of the data included in the fit. In addition a parameterisation uncertainty is evaluated by varying the functional form 

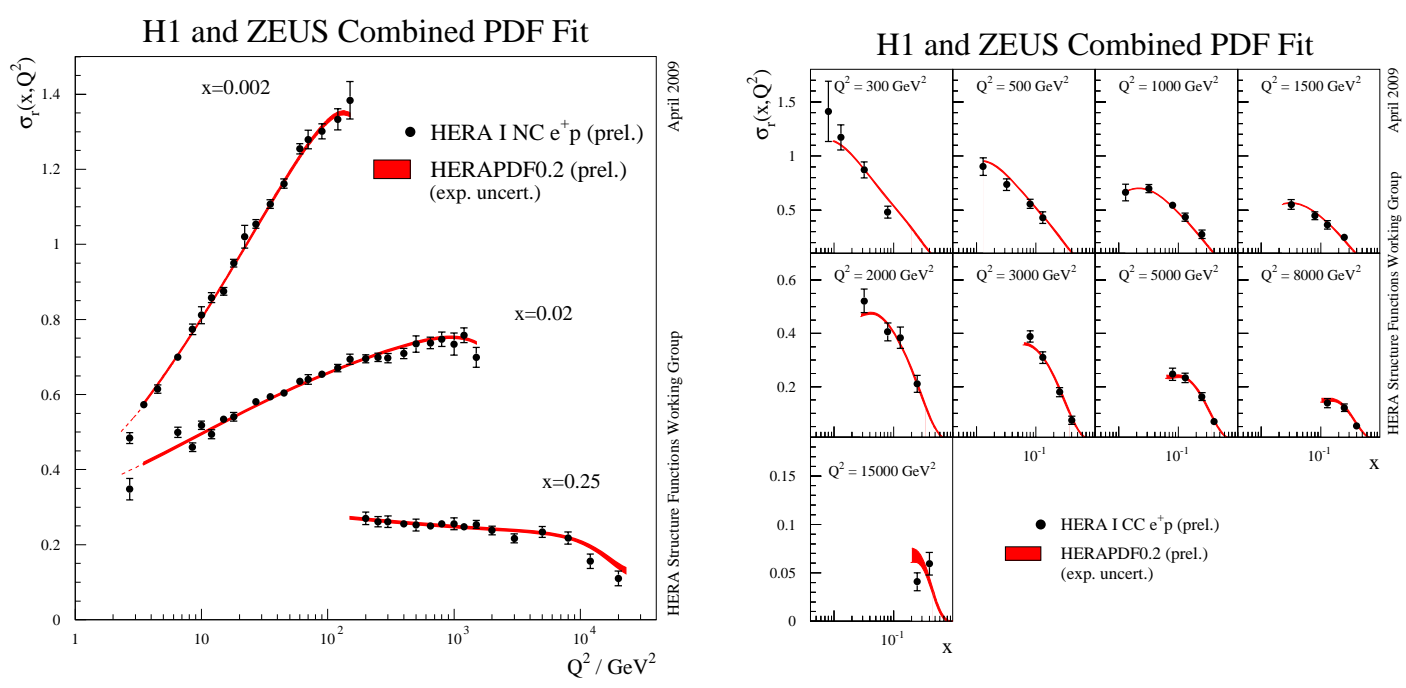

FIGURE 1. Selected measurements of the reduced NC cross section shown as a function of $Q^{2}$ (left). The reduced $\mathrm{CC} e^{+} p$ cross section shown as a function of $x$ (right). The measurements shown are combined H1 and ZEUS data. Also shown is the results of the NLO QCD fit to the data.

of the PDFs at the starting scale. The results of the fit are shown for $Q^{2}=10000 \mathrm{GeV}^{2}$ in figure 2. It can be seen that the sea and gluon densities are well constrained at low $x$. The valence quark densities are of a reasonable precision at medium $x$. The gluon is not well constrained at the highest $x$.

H1 and ZEUS Combined PDF Fit
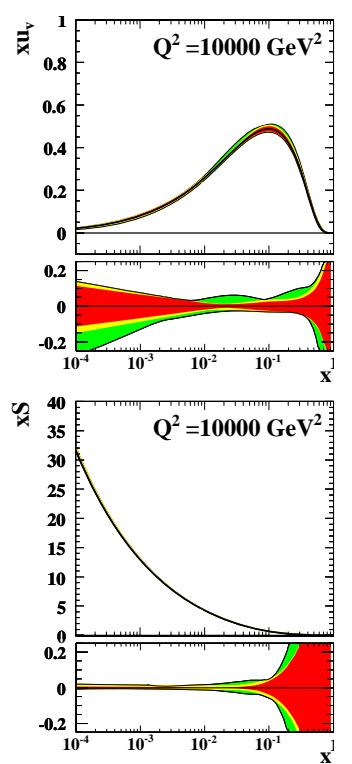
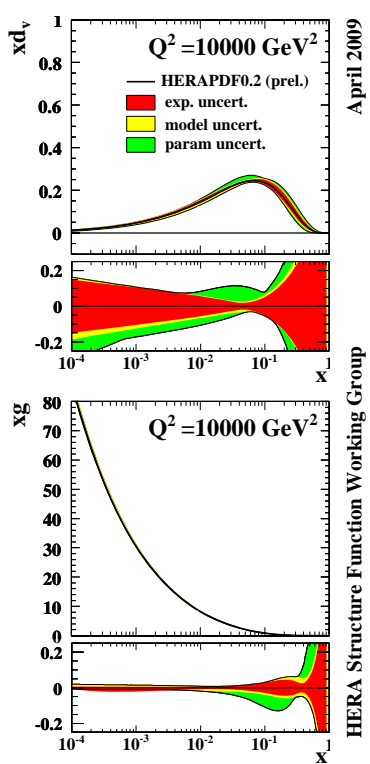

H1 and ZEUS Combined PDF Fit
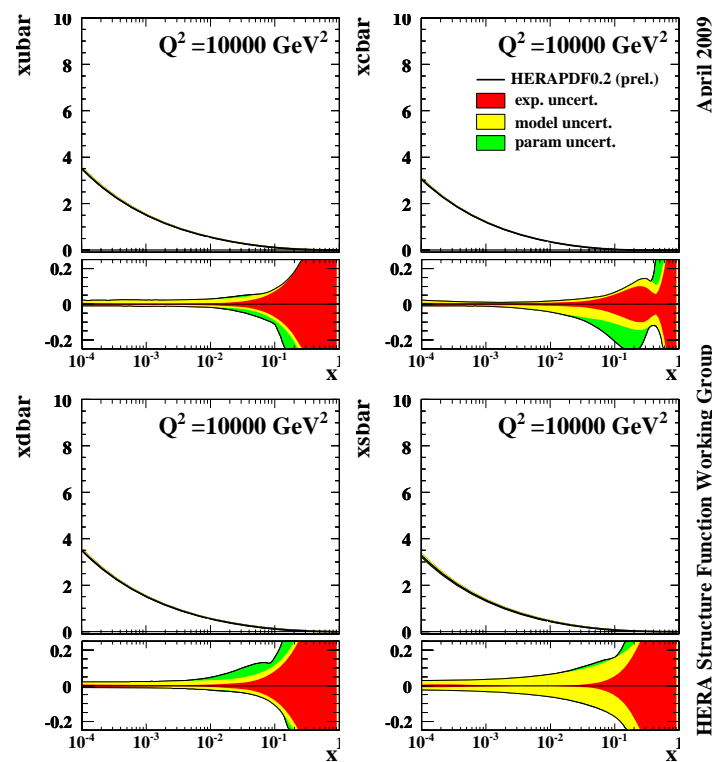

FIGURE 2. The PDFs together with their uncertainties extracted from the HERA data using an NLO QCD fit for $Q^{2}=10000 \mathrm{GeV}^{2}$. 
Measurements of $F_{L}$ have been made [5] by taking the difference of NC cross sections measured at the same $x$ and $Q^{2}$ but different $y$ (see equation1). The measurement from $\mathrm{H} 1$ is shown in figure 3 , along with QCD predictions. This $F_{L}$ data has not been used in the fits. The fits describe the data well at high $Q^{2}$ but tend to lie below the data at lower $Q^{2}$.

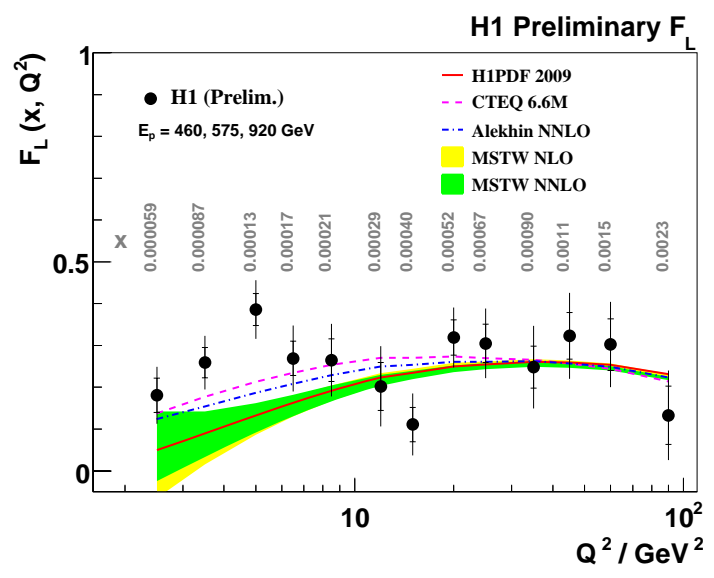

FIGURE 3. Measurement of the $F_{L}$ from $\mathrm{H} 1$ data shown as a function of $Q^{2}$. Also included are expectations from QCD.

\section{SUMMARY}

Recent measurements from HERA of the NC and CC cross sections and extracted structure functions have been presented. The precision of the data has been improved for many measurements by combining $\mathrm{H} 1$ and ZEUS data. The parton distribution functions of the proton have been extracted by fitting the HERA data with a QCD fit. The fit includes estimates of the experimental, model and parameterisation uncertainties.

\section{REFERENCES}

1. F.D. Aaron et al. [H1 Collaboration], Submitted to Eur. Phys. J.C., arXiv:0904.0929; F.D. Aaron et al. [H1 Collaboration], Submitted to Eur. Phys. J.C., arXiv:0904.3513.

2. H1 and ZEUS Collaborations, H1prelim-09-045, ZEUS-prel-09-011 (2009), http://www-h1.desy.de/h1/www/publications/htmlsplit/H1 prelim-09-045.long.html

3. H1 and ZEUS Collaborations, H1prelim-06-142, ZEUS-prel-06-022 (2006), http://www-h1.desy.de/h1/www/publications/htmlsplit/H1prelim-06-142.long.html

4. A. D. Martin, W. J. Stirling, R. S. Thorne and G. Watt, arXiv:0901.0002.

5. H1 Collaboration, H1prelim-08-042 (2008)

http://www-h1.desy.de/h1/www/publications/htmlsplit/H1 prelim-08-042.long.html H1 Collaboration, H1prelim-09-044 (2009)

http://www-h1.desy.de/h1/www/publications/htmlsplit/H1 prelim-09-044.long.html S. Chekanov et al. [ZEUS Collaboration], DESY-09-046 (2009), arXiv:0904.1092. 